\title{
Optimal design and control for UPFC using the evolutionary algorithms
}

\author{
M. Firouzjahi*1 and A. Shokri ${ }^{2}$ \\ ${ }^{1}$ Department of Mathematics, Payame Noor University, Iran \\ ${ }^{2}$ Iran Mines and Industries, Iran
}

\begin{abstract}
Among the Unified Power Flow Controller (UPFC) tools, Flexible Alternating Current Transmission Systems (FACTS) have ability to control the transmitted power, improve transient and dynamic stability and improve the profile of the voltage and damping of the oscillations in the power system. Using the proportional-integral (PI) and proportional-integral-derivative (PID) controllers is a custom method. Selecting the PI and PID coefficients is through different methods. Also designing a resistant controller which can control the system in different points of work has been continuously considered by researchers. In this regard, in order to improve the performance of UPFC controllers, adjusting its parameters is required optimally which this matter itself would facilitate accessing to control objectives. In this project, UPFC is used for damping the oscillations of the power system. Also, in order to adjust the controller parameters optimally, evolutionary algorithms like Ant Colony Optimization (ACO), Particle Swarm Optimization (PSO), Hybrid Particle Swarm Optimization (HPSO) and other algorithms are used.
\end{abstract}

Keywords: FACTS tools; UPFC; Damping of oscillations; Power system; Genetic algorithm

\section{Introduction}

Now a days, with development and advancement of aggregate power systems and daily increase in consumption of electric energy and reduction in the margin of stability in comparison to the past, the studies on stability of power systems have found more importance. Today, advances in the theory of dynamic and control systems, tools and strong methods of calculation and control systems and protection plans, have made it possible to recognize the different aspects of the problem of stability and its analysis and improvement (Parizad et al., 2009).

One of the main tools to control and conduct the power in a given path is the phase commutator device which has beeninvestigated since long time before considering the examined theory. The main application of commutator is to inject the series voltage with controlled amplitude and phase to the transfer line.The voltage is supplied by the excitation transformer. Given the slow performance, the ordinary phase commutators are not desirable for the transient state. During the recent years, another element called unified power flow controller (UPFC) has been proposed as an appropriate alternative which is a kind of FACTS tools and is very simi- lar to the phase commutator in terms of structure and performance (Payam et al., 2014).

Indeed, UPFC is one of the FACTS elements with multiple control objectives. The main objective of this element is to control the power flow. Controlling the voltage, improvingthe transient stability and damping of the oscillations in power system is among its other applications (Golkar et al., 2012). The incomparable ability of UPFC in controlling the transient power may be described well when the power system is subjected to dynamic turbulences which have the oscillations of the power. So long as UPFC controls the voltage of the sent terminal effectively, it has the capability to pass the desirable power from the transfer line under dynamic and persistent conditions (Barati, 2012).

In other words, most of the methods of designing the controllers have this assumption that they have a relatively accurate model of the accessible system. These models are usually obtained after linearizing the non-linear equations around the operating point. Designingthe ordinary stabilizers is based on the worst operating point in order to increase the moment of the damper. It is evident that if a change occurs

*Corresponding author. e-mail: Firouz1400@yahoo.com 
in the parameters of the model, the derived results would not have necessarily any validity. Perhaps, the comparative control is a selection for prosecuting the operating point for a satisfactory performance, butselecting an appropriate method for recognizing based on the number of parameters straight away and slow rate of operationalism are with some difficulties which do not exceed the simulation limit practically (Nayyeri et al, 2009). Hence, the aim of our study is to optimizecontroller design for UPFC using the evolutionary algorithms.

\section{Unified power flow controller (UPFC)}

FACTS technologies may have many positive influences on the validity of the power systems running. The real obtained advantages may be recognized through the appropriate modes and experiences. currentle emerging techniques for evaluating the composite power systems are mainly concentrated on the custom productions and facilities transferring the concentration (Komar, 2011).

In general, many types of FACTS controllers have been recognized throughout the worldamong which most important and known controllers are:

\section{Load Top Changer (LTC)}

Phase Angle Regulator

Static Var Compensation (SVC)

Static Compensation (STATCOM)

Unified Power Flow Controller (UPFC) (Panagis et al, 2007)

The concept of the unified power flow controller (natural load of the line) was offered by Gayougi in year 1991. UPFC was built for controlling the real time and dynamic compensation of AC transfer system in order to create required multi-functional flexibility for solving many problems faced by the industry of power transfer. UPFC is able to control all parameters influencing the power flow in the transfer line (i.e. voltage of impedance and phase angle) and this unique capability makes sense with the constraint of "unified" in its name. In addition, it can independentlycontrol the flow of both real and reactive powers in the control line (Abido, 2009).

As it was stated, UPFC is one of the index tools of flexible AC transfer systems having prominent capabilities for con- trolling the parameters of utilizing the transfer systems in steady state and also the capability to improve the dynamic behavior of the system in the transient state. No significant work has been carried out in the field of recognizing its dynamic behavior and capability to remove the oscillations of the power system. However, in this research, it is attempted to recognize the ability of this powerful element. UPFC is one of the index tools of AC transfer systems having prominent capabilities for controlling the parameters of utilizing the transfer systems in the steady state and also capability to improve the dynamic behavior of the system in the transient state (Khalili et al, 2011).

Indeed, the main application of UPFC in power systems is to control the active and reactive powers in the steady state of the power system, but due to high rate and the flexibility it canbe also used in particular rapid conditions. If in the transient state, the oscillations are not controlled in a way, the instability of the whole system will be resulted. According to the importance of this problem, the influence of using the UPFC in damping the oscillations in the transient state is of high importance. So that,the role and influence of UPFC in the stability of the power systems is investigated here along with considering a sample power system. From the viewpoint of an electrical engineer, the system is instable when the control systems do not cope with the distortion and the protection systems coming into practice to maintain the health of the system, although the generators and turbines have very strong protection systems (Norouzi, 2010).

Since UPFC may pass the considered power from the line either in permanent operating state or in dynamic conditions, the model of automatic load distribution control is used to remove the power of the network (Shalchi et al, 2013).

The parallel UPFC transformer of the series transducer may has active power exchanging with the network, which in this state, without change in the voltage of the DC capacitor, the additional active power required for the series transducer is provided through transformer and parallel transducer. Therefore, the series injection voltage phase may be controlled freely and independently. The ability of UPFC in controlling $\mathrm{P}$ and $\mathrm{Q}$ independently is very influential in neutralizing the dynamic distortions of the systems and balancing the active and reactive powers passing through the parallel 
lines. The control concept of UPFC according to the descriptions given in the first part of the controller is ability for voltage injection without any constraints and was discussed and called UPFC previously. UPFC makes controlling the passage of the power in different ways possible by adding the injected voltage phasor (Vpq with the amplitude of $\mathrm{Vpq}$ and angle) to Vs of the voltage phasor at the beginning of the line.

With appropriate selection of Vpq phasor, passage controlling or a combination of them may be created using three methods (Bourani et al, 2010).

1- Controlling the terminal voltage of UPFC: it may control the voltage like a top transformer with very small steps.

2- UPFC compensation control: it may also perform series compensation.

3- Phase shift control (transfer angle): phase shift by UPFC is in the state which finds phase change, but its amplitude does not change.

4- Multi-purpose control of UPFC: it may control the power passage at the same time through several methods. Compensation is carried out at the same time with controlling the terminal voltage, line-series compensation and adjustment of the phase shift angle.

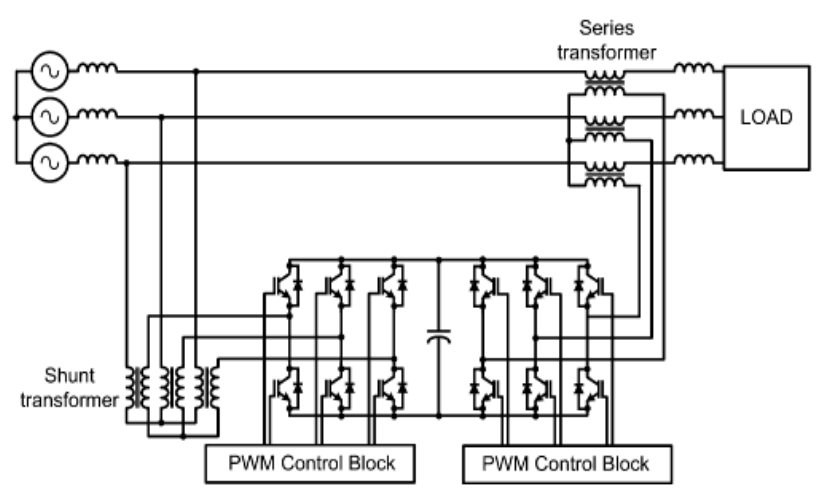

Fig. 1. UPFC power circuit

\section{Internal architecture of UPFC}

UPFC was presented to increase thepermittivity of power in the lines, improve the stability in the network and implementing the multi-purpose compensation. In addition, its application makes the controlling of all parameters related to the power relation (voltage, impedance and phase angle) possible. The facility for controlling the power in the allowable range of performance of UPFC and the network conditions is possible and theprotections of this equipment workin the conditions out of the nominal values of UPFC in order not to damage the equipment seriously. These limitations would be influential in the performance method of UPFC. Differentresponses of this equipment to the changes in the reference points in various control modes of these reference points is usually in the range of a few milliseconds. This appropriate rate increases the role of this equipment in the transient stability (Amara, 2012).

According to figure 2, the architecture of the UPFCincludes two transducers of voltage source (VSC); one of them is connected to the network through an excitement transformer (ET) in parallel, and another one is connected to the system in a series way through the injection transformer (BT). The DC side of both transducers is along with a joint capacitor. Every inverter uses the electronic power keyscapable of being turned off like IGBT or GTO. The output of each inverter is controllable in terms of size, phase and the produced voltage by both parameters of modulation of amplitude $(\mathrm{m})$ and modulation of phase angle $(\delta)$ and using the PWM switching algorithm.

The inverter of voltage source (1) as the static compensation (STATCOM), approximately injects sinusoidal flow with variable amplitude in the connection point.

The inverter of voltage source (2) as the static series compensation (SSSC) approximately injects sinusoidal voltage

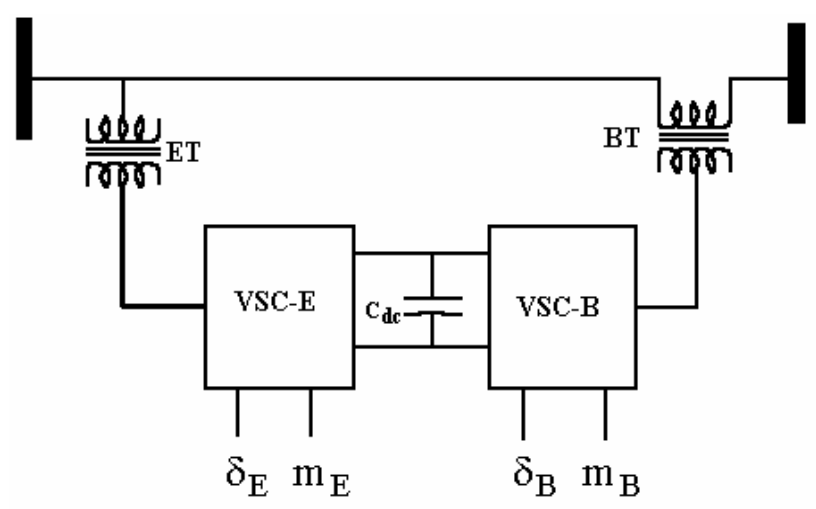

Fig. 2. Architecture of a UPFC 
with variable amplitude in the connection point. This injection voltage may have any angle with regard to the flow. Therefore, the exchange in active power in inverter terminals is possible through joint DC link capacitor.

Various control algorithms to control the FACTS tools in damping the oscillations of power

many recent researchers were planning to use more appropriate control methods with better time responses to control the FACTS tools in order to improve the stability of power systems which in this section, it is proceeded to introduce some of the used and suggested methods in the articles and comprise performances (Tivari, 2012).

\section{Proportional integral controller (PI)}

It is used for controlling the FACTS toolsas one of the most important and conventional controllers. In general, its basic techniques are very simple to run and logically influential and they have simple structure, easy design and low price, but this controller is extremely dependent on work point and it not suitable for non-linear applications and also their performance is inappropriate and destructive when a great distortion occurs in the network.

\section{Fuzzy logic controller (FLC)}

unlike PI controllers, these controllerscan lead togood results work point changes and they have less sensitivity than the PI controller. This controller is used in combination with the PI controller and the diagram block of the produced controller (fuzzy-p) is represented in figure (3) (Bourani et al, 2010).
PID controllers: the importance and position of PID controllers has encouraged many researchers to generalize the idea of PID controllers to multi-variable systems. A simple search in the indexed papers in this field, represents that only in the past decade, several hundred papers are published in the field of multivariable or multi-loop PIDs. The PID controllers have almost good resistance in facing the uncertainties, in addition to simplicity, low costs, efficiency and easiness of maintenance. Thousands of successful applications of these controllers in industrial systems have made their trace more firm as one of the most influential methods of designing the control systems. The proportional $(\mathrm{P})$ action of the output, adjusts the controller according to the size of error. The integral (I) action removes the error of the steady state, and the derivative (D) actionenters a type of prediction into the control. These three control actionsrectify the problem of control for many of the industrial processes, and the transparency of their performance ensures their use in practice. The PID controllersare used in two ways in multivariable systems. The first class designs the PID SIS controllers separately for every ring by disregarding the interference, although it is possible that each one of these rings to be adjusted well, but we know that closed-ring system has not necessarily any appropriate performance and efficiency, even instability of the closed-ring system is also possible. There is direct relation between the quality of performance of one-ring or non-concentrated PID controllersand the rate of interference and complexity of the multivariable system. The second class is multivariablePID control systems which are designed and adjusted by considering the interference and its influences. These controllers are concentrated and they can also control systems having severe interferences with correct design (Maqamianzadeh and Hassan, 2015).

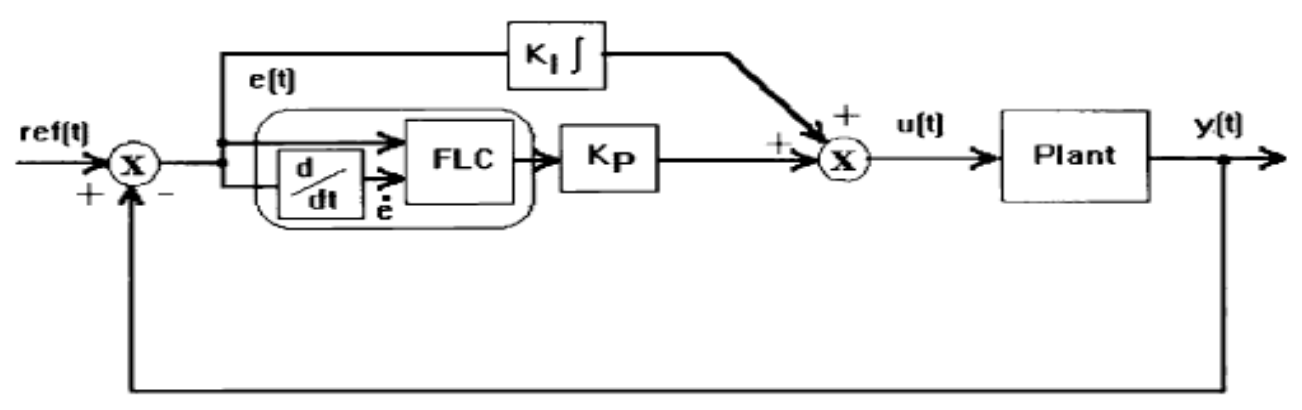

Fig. 3. Fuzzy controller 


\section{Methodology}

According to the investigated issue in the current research, the applied method is non-linear simulation. Hence, at first, we proceed to design the damper control for stability.

\section{Designing the damper controller for stability}

The damper controller is designed to produce an electric torque which has same phase with the changes in the velocity based on the method of phase compensation. The input of the damper controller is usually $\triangle \omega$ or $\triangle \delta$ and its output may be each one of the four input signals of UPFC, i. $m_{B}, m_{E}$, $\delta_{B}$ or $\delta_{E}$. In this research, $\triangle \omega$ is considered as the input of the damper controller and $\delta_{B} \omega$ is considered as the output of the damper controller. The structure of the damper controller is represented in figure 4 which includes a gain, a washout block and a phase compensation block. The optimized parameters controlling the damper are obtained using the genetic algorithm. Hence, before discussing about the problem of optimizing the controller, an introduction to the method of genetic algorithm optimization is presented.

\section{Optimizing the controller using genetic algorithm}

To find the coefficients of the damper controller constant (figure 1), in this research, the method of genetic algorithm optimization is used. In order to optimize the problem, the genetic algorithm may be considered binary and continuously and, continuous genetic algorithm is usedto solve this problem. Steps of optimization through this algorithm are as follows: optimized using genetic algorithm. Thus, there are three variables for optimization. The number of chromosomes is considered 48 in this research.

2- Cost function: in order to find the most optimized answer, the cost of each chromosome should be calculatedand the chromosomes with less cost are selected to produce new states and remaining states are removed from the initial matrix. when selectingthe most optimized controller parameters to improve the stability of the small signal and increase the damping of the system, the cost function is considered as following relation:

$$
J=\alpha \int t|\Delta \omega| \mathrm{d} t+\beta \int t\left|\Delta V_{D C}\right| \mathrm{d} t
$$

$\triangle \omega$ : changes in the system speed

$$
\triangle v_{\mathrm{DC}} \text { : changes in voltage }
$$

3- Producing new states and mutation: after calculating the cost of each chromosome using the cost function, the superior states or the states with less cost are selected for producing new states and the number of these superior states is determined by the maintenance rate. In this problem, the maintenance rate is selected to be 0.5 . Alsoto produce the new chromosomes from superior chromosomes, different methods are availableandfollowing relations are used for producing new states in this paper.

Father's c hromo some $=k_{L E}^{2 n}, T_{A}^{p / \alpha}, T_{B}^{p r t}$

Mother's chromos ome $=\left(K_{D i}^{m i x}, T_{\ell}^{m a}, T_{\Delta !}^{p i s}\right)$

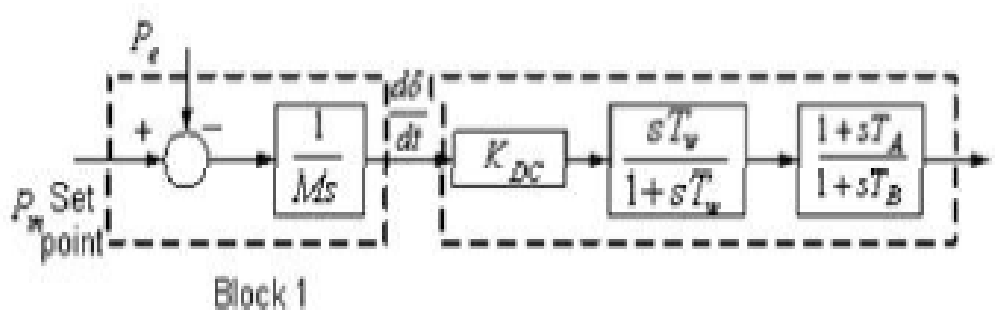

Fig. 4. Architecture of the damper controller

1- Formation of the initial population: the initial population is considered as a matrix in which everyline is called chromosome. In this problem, two constant controller coefficients of figure 4 are the variables which should be

$$
\begin{aligned}
K_{D C}^{n e w} & =\eta K_{D C}^{p a}+(1-\eta) K_{D C}^{m a}, 0 \leq \eta \leq 1 \\
T_{A}^{n e w} & =\eta T_{A}^{p a}+(1-\eta) T_{A}^{m a}, 0 \leq \eta \leq 1 \\
1 & T_{B}^{n e w}=\eta T_{B}^{P a}+(1-\eta) T_{B}^{m a}, 0 \leq \eta \leq 1 \quad \text { ller: the mentioned }
\end{aligned}
$$


controller has three parameters which in the allowable range, these parameters are selected such that they guarantee the stability of the system, and the objective is to obtain the most optimized value for the above parameters in the allowable limit. The results of this optimized search is as below:

$\mathrm{K}_{\mathrm{DC}}=631.6$

$\mathrm{T}_{\mathrm{A}}=0.25$

$\mathrm{T}_{\mathrm{B}}=0.1$

\section{Results and discussion}

In order to represent the influence of the optimized damper controller in comparison to the classic controller, a classic damper controller is designed using the linear model with the phase compensation method which the parameters of this controller are as below:

$\mathrm{K}_{\mathrm{DC}}=570$

$\mathrm{T}_{\mathrm{A}}=0.029$

$\mathrm{T}_{\mathrm{B}}=0.1$

After applying the above controllers to the system, the results of non-linear simulation of the system is carried out per applying the input of the step with the amplitude of 0.1 to the input of the reference mechanical power and also three phase short circuit on the terminal of the generator which its results are represented in figs. 5, 6 and 7. Also,to take into account the influence of increasing the load on the network, the system load has increased from a nominal load to a heavy

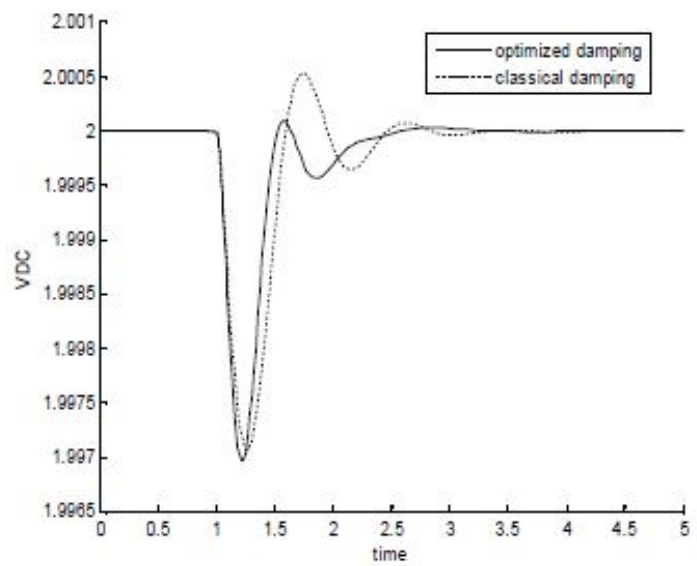

Fig. 5. $v_{D C}$ curve per applying the step with the amplitude of 0.1 to the input of the reference power $T_{m}$ load and then, the results of short circuit of the generator terminal for every heavy load are also represented in figures 8 and 9.

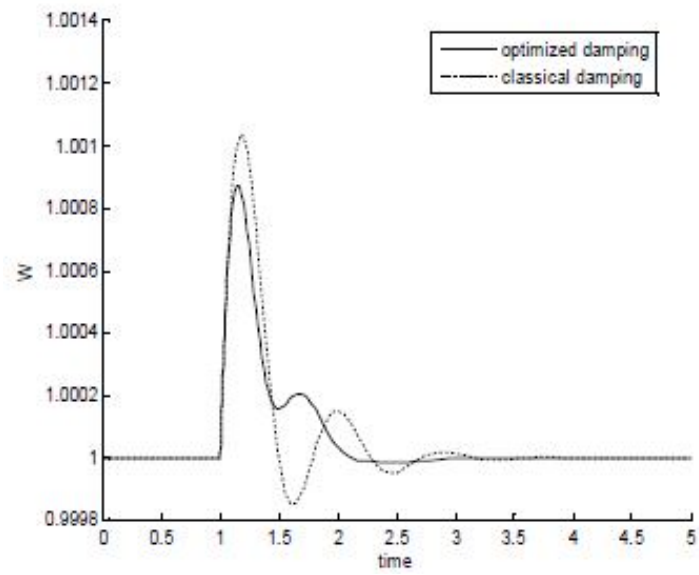

Fig. 6. $\omega$ curve per applying the step with the amplitude of 0.1 to the input of the reference power $T_{m}$

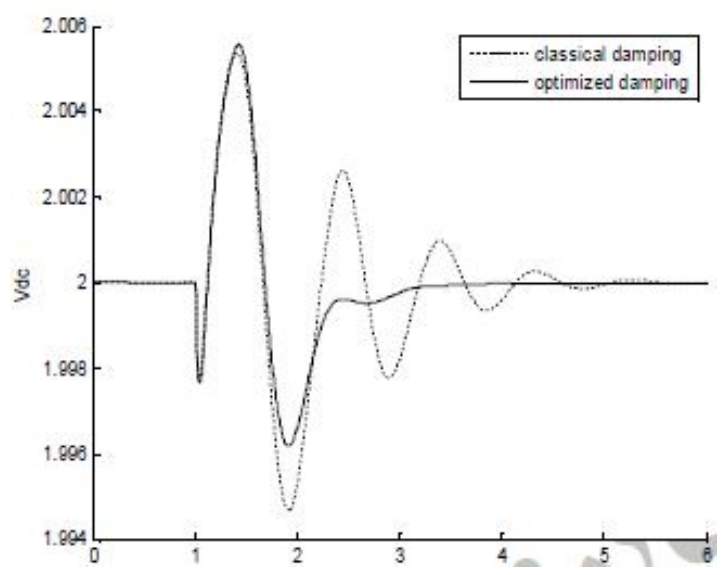

Fig. 7. $v_{\mathrm{DC}}$ curve per short circuit in the terminal of the generator for 17 milliseconds in the nominal load

As it is observed, the response of system with optimized damper controller is more appropriate than the classic controller.The results represent that the controller which is designed through genetic algorithm is more appropriate than the classic controllers against the turbulences of small signal type. 


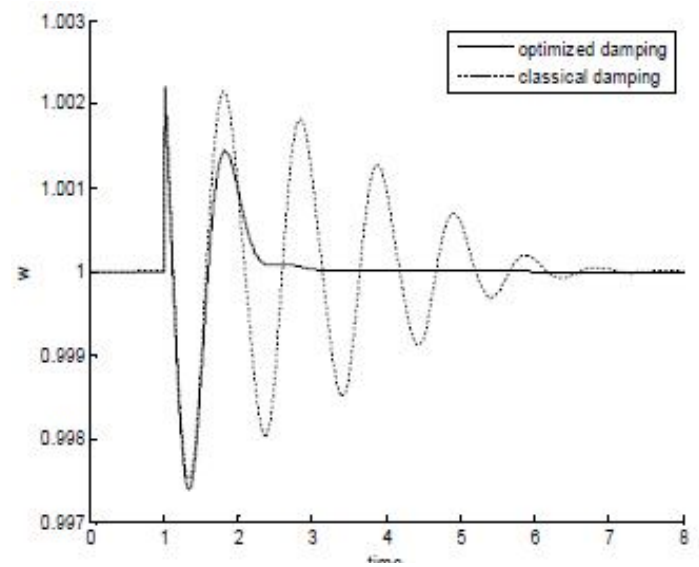

Fig. 8. $\omega$ curve per short circuit in the terminal of the generator for 17 milliseconds in heavy load

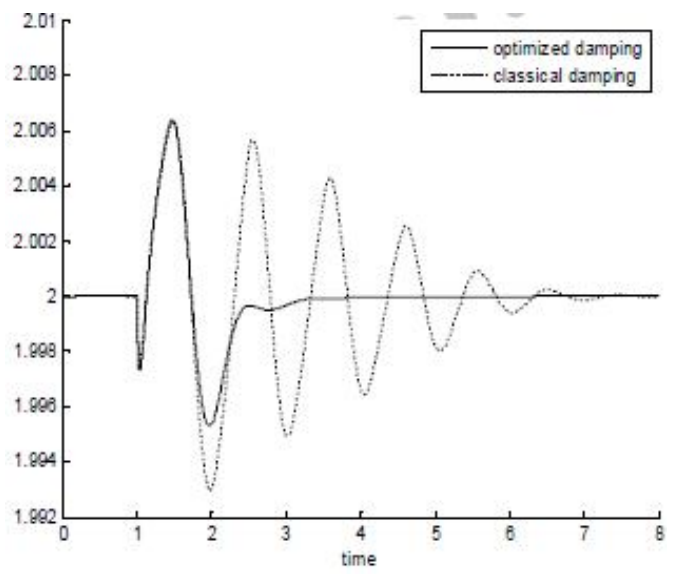

Fig. 9. $\omega$ curve per short circuit in the terminal of the generator for 17 milliseconds in heavy load

\section{Conclusion}

The purpose of applying the FACTS tools in transfer and distribution systems isto rectifysome problems including achievea high quality in power distribution networks, increase in chargeability of power systems and management of line fulfillment. As mentioned, one of these tools is UPFC which in addition to above mentionedfunctions; it also can affect the connected network: for instance,improving the oscillations of the power angles and damping the oscillations and increasing the stability of the passage. Generally, in this research, the results of using the damper controller optimized through the genetic algorithm explains the influence of this controller on stabilizing the system. Moreover, the controller of the mentioned damper, well damps the oscillations of the system when slight distortions like changes of the mechanical power enter into the generator or great distortions like short circuit in the system, and in the state of stability of slight signal and great signal, it has more appropriate response than the damper controller designed through classic method.

\section{References}

Abido MA (2009), Power System Stability Enhancement Using FACTS Controllers: A review, The Arabian Journal for Science and Engineering, 34, Number 1B.

Amara S (2012), Power system stability improvement by FACTS devices: A comparison between STATCOM, SSSC and UPFC, Renewable Energies and Vehicular Technology (REVET), 2012 First International Conference on IEEE, pp. 360 - 365.

Barati H (2012), the intellectual control of the unified power flow controller (UPFC) in order to improve the transient stability in multi-machine power systems, journal of investigation in the technology of the electricity, 2nd year, no. 2

Bevrani H, Ghosh A, Ledwich G (2010), Renewable Energy Sources and Frequency Regulation: Survey and new perspectives IET Renew. Power Gener., 4(5): 438-457.

Golkar, Masoud Ali Akbar, Mahi, Abdulkarim, 2012. A new method for controlling D-UPFC in the distribution networks without delay, the papers of Iranian congresses. $13^{\text {th }}$ global conference of networks of distributing the electricity.

Khalili, Mohammad; Sadegh Ahmadian and Mohammad Hosein Kazemi, (2011), Design of fuzzy-neural controller of multi-machine power system based on the comparative control and comparing it with the classic controllers, $25^{\text {th }}$ international conference of electricity, Tehran, Tavanir co., Niroo research institute.

Maqamianzadeh M and Hassan B (2015), Investigation on the performance of UPFC and IPFC in restructured 
power systems in order to maximize the social welfare and management of fulfillment, 1st regional congress on the information technology of the electricity of refinement, Gachsaran, scientific-applicable center of Gachsaran.

Nayyeri Pour, Majid, Yazdian Verjani, Ali, Mohammadian, Mostafa (2009), design of unified power flow controller in bilinear way in order to improve the stability of the network, journal of electrical engineering, 6th year, no. 3

Norouzizadeh, Reza, (2010), designing and simulating the unified power flow controller using the matrix transducer, Shahroud university of technology.

Panagis N, Vovos Aristides E, Kiprakis A, Robin Wallace, and Gareth P Harrison (2007), Centralized and Distributed Voltage Control: Impact on Distributed Generation Penetration, IEEE Transactions on Power Systems, VOL. 22, NO. 1.

Parizad A, Khazali A, Kalantar M (2009), Application of HSA and GA in optimal placement of FACTS devices considering voltage stability and losses. International Conference on Electric Power and Energy Conversion Systems (EPECS '09), pp. 1-7, 10-12 Nov.
Payam, Mohammad Sadegh, Saeed Abazari and Noushin Shahcheraghi (2014), Application of the method of controlling the sliding mode for controlling the UPFC with the aim of improving the transient stability in onemachine power system, 1st national congress on Iranian electrical engineering, Bandar-e Gaz, Islamic Azad university of Bandar-e Gaz.

Shalchi, Farshid, Shayeqi, Hosein, Shayanfar, Heydar Ali, (2013), design of resistant controller for UPFC, 26th international conference on the electricity.

Sheela T (2012), Neural network predictive control of UPFC for improving transient stability performance of power system", Applied Soft Computing Journal, 11(8): 4581-4590.

Received: 01 February 2016; Revised: 03 March 2016; Accepted: 24 April 2016. 